\title{
TRANSLATION REPRESENTATIONS FOR AUTOMORPHIC SOLUTIONS OF THE WAVE EQUATION IN NON-EUCLIDEAN SPACES; THE CASE OF FINITE VOLUME
}

\author{
BY
}

PETER D. LAX AND RALPH S. PHILLIPS ${ }^{1}$

\begin{abstract}
Let $\Gamma$ be a discrete subgroup of automorphisms of $\mathbf{H}^{n}$, with fundamental polyhedron of finite volume, finite number of sides, and $N$ cusps. Denote by $\Delta_{\Gamma}$ the Laplace-Beltrami operator acting on functions automorphic with respect to $\Gamma$. We give a new short proof of the fact that $\Delta_{\Gamma}$ has absolutely continuous spectrum of uniform multiplicity $N$ on $\left(-\infty,((n-1) / 2)^{2}\right)$, plus a standard discrete spectrum. We show that this property of the spectrum is unchanged under arbitrary perturbation of the metric on a compact set. Our method avoids Eisenstein series entirely and proceeds instead by constructing explicitly a translation representation for the associated wave equation.
\end{abstract}

Introduction. Using the methods developed in Parts I and II of [5], we obtain a short proof for the existence and completeness of incoming and outgoing translation representations for the wave equation acting on automorphic functions with fundamental polyhedron of finite volume and a finite number of sides. As a by-product we show that the associated Laplace-Beltrami operator has a standard discrete spectrum plus an absolutely continuous spectrum of uniform multiplicity $N$ on $(-\infty$, $\left.((n-1) / 2)^{2}\right)$, where $n$ is the dimension of the hyperbolic space and $N$ the number of cusps. We also treat automorphic solutions of a perturbed wave equation when the support of the perturbation is compact.

A spectral theory for the Laplace-Beltrami operator in this setting was first obtained by A. Selberg in his seminal paper [7] of 1956. Since then several different approaches to this problem have been developed among which we note that of Faddeev [1] and Faddeev and Pavlov [2]. The latter paper made the connection with the Lax-Phillips theory of scattering which we exploited in our 1976 monograph [3]. All of these treatments arrived at the spectral theory via the Eisenstein functions. In the present paper we attack the problem directly through the translation representation, which can be given by an explicit integral formula; this method was first described in [6]. A more complete discussion of this problem can be found in Venkov [8].

Received by the editors July 6, 1984.

1980 Mathematics Subject Classification. Primary 30F40, 35J15, 47A70; Secondary 10D15.

${ }^{1}$ The work of the first author was supported in part by the Department of Energy under contract DE-AC02-76 ERO 3077 and the second author by the National Science Foundation under Grant MCS-8304317 and the Danish National Science Research Council under Grant 11-3601. 
1. Elementary properties of the translation representations. Let $\Gamma$ be a discrete group of motions in $\mathbf{H}^{n}$, having a fundamental polyhedron $F$ with a finite number of sides as well as a finite volume. Thus $F$ can have only cusps of maximal rank and no free sides. We denote the $L_{2}$ and Dirichlet integrals by $H$ and $D$ :

$$
H_{F}(u)=\int_{F}|u|^{2} \frac{d x d y}{y^{n}}, \quad D_{F}(u)=\int_{F}\left(\left|u_{x}\right|^{2}+\left|u_{y}\right|^{2}\right) \frac{d x d y}{y^{n-2}} .
$$

Here we use the Poincaré upper half-space to model the real hyperbolic space $\mathbf{H}^{n}$.

We define the operator $L$ to be the Laplace-Beltrami operator plus $((n-1) / 2)^{2}$;

$$
L=y^{2}\left(\partial_{y}^{2}+\Delta_{x}\right)-(n-2) y \partial_{y}+\left(\frac{n-1}{2}\right)^{2} .
$$

Our main tool is the corresponding non-Euclidean wave equation

$$
u_{t t}=L u \text {. }
$$

The energy associated with this equation is

$$
\begin{aligned}
E_{F}(u) & =-H_{F}(u, L u)+H_{F}\left(u_{t}\right) \\
& =D_{F}(u)-\left(\frac{n-1}{2}\right) H_{F}(u)+H_{F}\left(u_{t}\right) .
\end{aligned}
$$

Energy is conserved with the passage of time by solutions of the wave equation.

When $\operatorname{vol}(F)<\infty, E_{F}$ always takes on negative values. However, this can be compensated for by adding a term of the form

$$
K(u)=c \int_{S}|u|^{2} d w=c H_{S}(u)
$$

where $S$ is a compact subset of $F$.

LEMMA 1.1. The form

$$
G=E_{F}+K
$$

is positive definite provided the constant $c$ and the compact set $S$ in (1.4) are large enough.

Proof. We decompose $F$ into cusp neighborhoods $N_{j}$ and a compact set $S$ :

$$
S=F \backslash \bigcup N_{j}
$$

$N_{j}$ is defined as follows: Map the $j$ th cusp into $\infty$; the resulting cusp neighborhood will be of the form

$$
N_{j}=F_{j} x(a, \infty)
$$

for $a$ sufficiently large, where $F_{j}$ is the compact cross-section of the transformed cusp. Next we perform an integration by parts:

$$
\begin{aligned}
\int_{a}^{\infty} y\left|\partial_{y}\left(\frac{u}{y^{(n-1) / 2}}\right)\right|^{2} d y & =\int_{a}^{\infty}\left(\frac{\left|u_{y}\right|^{2}}{y^{n-2}}+\left(\frac{n-1}{2}\right)^{2} \frac{|u|^{2}}{y^{n}}-\frac{n-1}{2} \frac{\partial_{y}|u|^{2}}{y^{n-1}}\right) d y \\
& =\int_{a}^{\infty}\left(\frac{\left|u_{y}\right|^{2}}{y^{n-2}}-\left(\frac{n-1}{2}\right)^{2} \frac{|u|^{2}}{y^{n}}\right) d y+\frac{n-1}{2} \frac{|u(a)|^{2}}{a^{n-1}} .
\end{aligned}
$$


Integrating over $F_{j}$ we get

$$
\begin{aligned}
\int_{F_{j}} \int_{a}^{\infty}\left|u_{y}\right|^{2} & \frac{d y d x}{y^{n-2}}-\left(\frac{n-1}{2}\right)^{2} H_{N_{j}}(u) \\
& =\int_{F_{j}} \int_{a}^{\infty} y\left|\partial_{y}\left(\frac{u}{y^{(n-1) / 2}}\right)\right|^{2} d y d x-\left(\frac{n-1}{2}\right) \int_{F_{j}} \frac{|u(x, a)|^{2}}{a^{n-1}} d x .
\end{aligned}
$$

To estimate the last term on the right we set

$$
u(a)=\int_{\eta}^{a} u_{y} d y+u(\eta), \quad a-1 \leqslant \eta \leqslant a
$$

by the Schwarz inequality

$$
|u(a)|^{2} \leqslant 2 \int_{a-1}^{a}\left|u_{y}\right|^{2} \frac{d y}{y^{n-2}} \frac{a^{n-1}-(a-1)^{n-1}}{n-1}+2|u(\eta)|^{2} .
$$

Integrating with respect to $\eta$ from $(a-1)$ to $a$ and dividing by $a^{n-1}$, we get

$$
\frac{|u(a)|^{2}}{a^{n-1}} \leqslant \frac{2\left(a^{n-1}-(a-1)^{n-1}\right)}{(n-1) a^{n-1}} \int_{a-1}^{a}\left|u_{y}\right|^{2} \frac{d y}{y^{n-2}}+2 a \int_{a-1}^{a}|u|^{2} \frac{d y}{y^{n}} .
$$

Integrate with respect to $x$ over $F_{j}$; for sufficiently large $a$

$$
\left(\frac{n-1}{2}\right) \int_{F_{j}} \frac{|u(x, a)|^{2}}{a^{n-1}} d x \leqslant \frac{1}{2} \int_{a-1}^{a} \int_{F_{j}}\left|u_{y}\right|^{2} \frac{d x d y}{y^{n-2}}+a(n-1) \int_{a-1}^{a} \int_{F_{j}}|u|^{2} \frac{d x d y}{y^{n}} .
$$

Combining (1.7) and (1.8) and summing over the cusps, we see that

$$
\begin{aligned}
D_{F}(u)-\left(\frac{n-1}{2}\right)^{2} H_{F}(u) \geqslant & \sum_{j=1}^{N} \int_{a_{j}}^{\infty} \int_{F_{j}}\left[y\left|\partial_{y}\left(\frac{u}{y^{(n-1) / 2}}\right)\right|^{2}+\frac{\left|u_{x}\right|^{2}}{y^{n-2}}\right] d x d y \\
& +\frac{1}{2} D_{S}(u)-c^{\prime} H_{S}(u),
\end{aligned}
$$

where $c^{\prime}=((n-1) / 2)^{2}+a(n-1)$. The assertion (1.5) follows if we set $c=c^{\prime}+1$.

We now complete the set of all $C_{0}^{\infty}(F)$ data with respect to the $G$ norm and denote the resulting space by $\mathscr{H}_{G}$. It is easy to show, by an argument similar to that used in the proof of Lemma 1.1, that for $f$ in $\mathscr{H}_{G}$ the local $L_{2}$ and Dirichlet norms of $f_{1}$ are majorized by $G(f)$. Hence it follows from Rellich's compactness theorem that $K$ is compact with respect to $G$. We list two direct consequences of this fact.

LEMMA 1.2. (a) $E_{F}$ is positive definite on a subspace of $\mathscr{H}_{G}$ of finite codimension.

(b) The positive spectrum of $L$ consists of a finite number of points $\lambda_{1}^{2}, \ldots, \lambda_{m}^{2}$ with corresponding eigenfunctions $\varphi_{1}, \ldots, \varphi_{m}$;

$$
L \varphi_{j}=\lambda_{j}^{2} \varphi_{j},
$$

where $\varphi_{j}$ is automorphic and square integrable, and $\lambda_{j}>0$.

Proof. Since $K$ is compact with respect to $G$, there is a subspace of finite codimension in which $K(f) \leqslant \frac{1}{2} G(f)$. On this subspace

$$
E(f)=G(f)-K(f) \geqslant \frac{1}{2} G(f) \text {. }
$$


This proves (a). Next suppose that $L$ is positive on a subspace $\mathscr{J}$ of $L_{2}(F)$. For $\varphi$ in $\mathscr{J}, H_{F}(L \varphi, \varphi)$ is finite and an integration by parts shows that $D_{F}(\varphi)<\infty$. Thus $f=\{\varphi, 0\}$ belongs to $\mathscr{H}_{G}$ and, by $(1.3), E_{F}(f)<0$. Part (a) of the lemma requires $\mathscr{J}$ to be finite dimensional and it follows from this that the positive spectrum of $L$ is pure point and finite.

The functions $e^{ \pm \lambda, t} \varphi_{j}$ are automorphic solutions of the wave equation. Their initial data

$$
p_{j}^{ \pm}=\left\{\varphi_{j}, \pm \lambda_{j} \varphi_{j}\right\}
$$

span a space which we denote by $\mathscr{P}$. A simple calculation shows that

$$
\begin{aligned}
& E_{F}\left(p_{j}^{+}, p_{k}^{+}\right)=0, \quad E_{F}\left(p_{j}^{-}, p_{k}^{-}\right)=0 \text { for all } j, k ; \\
& E_{F}\left(p_{j}^{+}, p_{k}^{-}\right)= \begin{cases}0 & \text { for } j \neq k, \\
-\lambda_{j}^{2} H_{F}\left(\varphi_{j}\right) & \text { for } j=k .\end{cases}
\end{aligned}
$$

It follows from these expressions that the energy form is nondegenerate in $\mathscr{P}$; so there is an $E$-orthogonal projection $P$ of $\mathscr{H}_{G}$ onto $\mathscr{P}$. We denote the complement $I-P$ by $Q$ and denote $Q \mathscr{H}_{G}$ by $\mathscr{H}_{Q}$.

Lemma 1.3. $E_{F}$ is nonnegative on $\mathscr{H}_{Q}$ and the null space $\mathscr{Z}$ of $E_{F}$ on $\mathscr{H}_{Q}$ is finite dimensional. Modulo this null space $\mathscr{H}_{Q}$ is complete with respect to $E_{F}$.

Proof. For $f=\left\{f_{1}, f_{2}\right\}$ in $\mathscr{H}_{Q}, f_{1}$ in the domain of $L$, both components are orthogonal to the $\varphi_{j}$. It follows in particular that $\left(L f_{1}, f_{1}\right) \leqslant 0$ so that, by (1.3), $E_{F}(f) \geqslant 0$. Since $C_{0}^{\infty}(F)$ data are dense in $\mathscr{H}_{G}$, it is easy to see that any data in $\mathscr{H}_{Q}$ can be approximated by data of this kind. That $\mathscr{Z}$ is finite dimensional follows directly from Lemma 1.2(a).

Now $\mathscr{H}_{Q}$ is a closed subspace and so it is the $G$-orthogonal complement $\mathscr{H}_{1}$ of $\mathscr{Z}$ in $\mathscr{H}_{Q}$. Any $f$ in $\mathscr{H}_{Q}$ can be decomposed into $G$-orthogonal parts: $f=z+g, z \in \mathscr{Z}$ and $g \in \mathscr{H}_{1}$. Since $z$ belongs to the null space of $E_{F}$ we have $E_{F}(f)=E_{F}(g)$. Thus the mapping $f \rightarrow g$ is an isometry in the $E_{F}$ norm. To prove the completeness of $\mathscr{H}_{Q} / \mathscr{Z}$ in the $E_{F}$ norm, it therefore suffices to prove that $E_{F}$ and $G$ are equivalent norms on $\mathscr{H}_{1}$. Obviously $E_{F} \leqslant G$ on $H_{1}$. Suppose next that the opposite inequality is not true; then there is a subsequence $\left\{f_{n}\right\} \subset \mathscr{H}_{1}$ such that

$$
G\left(f_{n}\right) \equiv 1 \text { and } E_{F}\left(f_{n}\right) \rightarrow 0 .
$$

Since $K$ is compact with respect to $G$ we can find a subsequence (which we renumber) such that $K\left(f_{n}-f_{m}\right) \rightarrow 0$. Since $E_{F} \geqslant 0$ in $\mathscr{H}_{1}$, we see from (1.12) that $E_{F}\left(f_{n}-f_{m}\right) \rightarrow 0$ and it follows that the $\left\{f_{n}\right\}$ form a Cauchy sequence in the $G$ norm. Thus $f_{n}$ converges to some $f$ in $\mathscr{H}_{1}$ which by (1.12) is of $G$ norm 1 . At the same time we see by (1.12) that $E_{F}(f)=0$; i.e. $f$ belongs to both $\mathscr{H}_{1}$ and its $G$-orthogonal complement $\mathscr{Z}$. This contradiction proves the last assertion in Lemma 1.3.

In what follows, the $E_{F}$ seminorm will be employed exclusively in $\mathscr{H}_{Q}$. Thus orthogonality, isometry, etc. are all to be taken with respect to $E_{F}$. Of course for isometry to be meaningful we must limit ourselves to cosets modulo $\mathscr{Z}$. 
Denote by $U(t)$ the solution operator relating initial data of solutions of the wave equation to their data at time $t$ :

$$
U(t)\left\{u(0), u_{t}(0)\right\}=\left\{u(t), u_{t}(t)\right\}
$$

Since energy is conserved, the operators $U(t)$ are unitary on $\mathscr{H}_{Q} / \mathscr{Z}$. They form a one-parameter group whose generator $A$,

$$
A=\left(\begin{array}{ll}
0 & I \\
L & 0
\end{array}\right)
$$

is skew-selfadjoint. It is obvious from (1.9) and (1.10) that the $p_{j}^{ \pm}$are eigenvectors of A :

$$
A p_{j}^{ \pm}= \pm \lambda_{j} p_{j}^{ \pm}
$$

It follows that $\mathscr{P}$ is an invariant subspace of $U(t)$ and hence so is its orthogonal complement $\mathscr{H}_{Q}$. As a consequence $Q$ commutes with $U(t)$ :

$$
Q U(t)=U(t) Q \text {. }
$$

Next we recall the definition of the incoming and outgoing translation representations $R_{-}^{F}$ and $R_{+}^{F}$. Each has $N$ components, one for each cusp, defined in terms of integrals over horospheres. To describe the $j$ th component $R^{j}{ }_{ \pm}$it is again convenient to map the $j$ th cusp into $\infty$, in which case the neighborhoods of the $j$ th cusp are of the form $(1.6)_{j} ; F_{j}$ is a compact fundamental polyhedron of the subgroup $\Gamma_{j}$ of $\Gamma$ keeping $\infty$ fixed.

With the $j$ th cusp at $\infty$ and $f=\left\{f_{1}, f_{2}\right\}$ the given automorphic data, we define

$$
R_{ \pm}^{j} f(s)=\frac{1}{\sqrt{2}}\left[\partial_{s} e^{(1-n) / 2} \overline{f_{1}}\left(e^{s}\right) \mp e^{(1-n) s / 2} \overline{f_{2}}\left(e^{s}\right)\right]
$$

where the bar denotes the mean value:

$$
\bar{f}(y)=\left|F_{j}\right|^{-1 / 2} \int_{F} f(x, y) d x .
$$

It is easy to verify that $R_{ \pm}^{j}$ is a linear transformation, mapping $\mathscr{H}_{G}$ continuously into $L_{2}(a, \infty)$ for each $a$ in $\mathbf{R}$.

LEMMA 1.4. $R_{ \pm}^{F}$ transmutes the action of $U$ into translation:

$$
\begin{aligned}
& R_{+}^{F} U(t) f=T(t) R_{+}^{F} f, \\
& R_{-}^{F} U(t) f=T(-t) R_{-}^{F} f
\end{aligned}
$$

here $T(t)$ denotes translation to the right by $t$ units.

REMARK. Because of properties $(1.18)_{ \pm}$we call $R_{+}^{F}$ a translation representation and $R_{-}^{F}$ an anti-translation representation.

Proof. Let $u(x, y, t)$ be a solution of the wave equation (1.2) with automorphic initial data $f=\left\{f_{1}, f_{2}\right\}: u(0)=f_{1}, u_{t}(0)=f_{2}$. The mean value $\bar{u}(y, t)$ will then satisfy the equation

$$
\bar{u}_{t t}=y^{2} \bar{u}_{y y}-(n-2) \bar{u}_{y}+\left(\frac{n-1}{2}\right)^{2} \bar{u} \text { for all } y>0
$$


The change of variables $s=\log y$ and $v=\bar{u} / y^{(n-1) / 2}$ transform (1.19) into the classical wave equation

$$
v_{t t}=v_{s s}
$$

and the initial data goes over into

$$
v(0)=e^{(1-n) s / 2} \overline{f_{1}}\left(e^{s}\right), \quad v_{t}(0)=e^{(1-n) s / 2} \overline{f_{2}}\left(e^{s}\right) .
$$

Setting

$$
k_{+}(s, t)=v_{s}-v_{t},
$$

it follows from (1.17) and (1.21) that

$$
k_{+}(s, 0)=\sqrt{2} R_{+}^{j} f .
$$

Since the solution at time $t$ is $U(t) f$, we have similarly

$$
k_{+}(s, t)=\sqrt{2} R_{+}^{j} U(t) f .
$$

It follows from (1.20) and (1.21) that $\partial_{t} k_{+}+\partial_{s} k_{+}=0$ and hence that

$$
k_{+}(s, t)=k_{+}(s-t, 0) \text {. }
$$

Combining (1.22) and (1.23) we obtain the assertion of the lemma for $R_{+}^{j}$ and hence for $R_{+}^{F}$. A similar argument with

$$
k_{-}(s, t)=v_{t}+v_{s}
$$

proves (1.18).

The isometry of $R_{+}^{F}\left(R_{-}^{F}\right)$ is easy to establish on what we call outgoing (incoming) data. These are defined as follows: Let $l$ be any $C_{0}^{\infty}$ function and set $m$ equal to its indefinite integral:

$$
m(s)=\int_{-\infty}^{s} l(r) d r .
$$

As we have seen in the proof of Lemma 1.4, the functions

$$
u_{ \pm}(y, t)=y^{(n-1) / 2} m(\log y \mp t)
$$

are solutions of the wave equations $(1.2)^{\prime}$. Being independent of $x$, they are automatically automorphic with respect to $\Gamma_{j}$.

Suppose $a_{j}$ is so large that the subset of $F$ (with the $j$ th cusp at $\infty$ ), consisting of those points whose $y$ coordinate is $>a_{j}$, belongs entirely to the $j$ th cusp; i.e. is of the form

$$
N_{j}=F_{j} \times\left(a_{j}, \infty\right) .
$$

Then if the support of $l$ is contained in $s>\log a_{j}, u_{+}$is automorphic with respect to the entire group $\Gamma$ for all $t \geqslant 0$, and $u_{-}$is likewise automorphic with respect to $\Gamma$ for all $t \leqslant 0$.

We now define the incoming and outgoing subspaces $\mathscr{D}_{-}^{j}$ and $\mathscr{D}_{+}^{j}$ as the initial data of all such solutions $u_{-}$and $u_{+}$, respectively, i.e. $\mathscr{D}_{-}^{j}$ consists of data of the form

$$
d_{-}=\left\{y^{(n-1) / 2} m(\log y), y^{(n-1) / 2} l(\log y)\right\}
$$

and $\mathscr{D}_{+}^{j}$ consists of data of the form

$$
d_{+}=\left\{y^{(n-1) / 2} m(\log y),-y^{(n-1) / 2} l(\log y)\right\},
$$


where $m$ and $l$ are related by (1.24) and $l$ is supported in

$$
s>\log a_{j} .
$$

It follows from (1.27) and (1.28) that $d_{+}$and $d_{-}$in $\mathscr{D}_{ \pm}^{j}$ are supported in $N_{j}$. We denote by $\mathscr{D}_{ \pm}$the sun of the $\mathscr{D}^{j}$ :

$$
\mathscr{D}_{+}=\mathscr{D}_{+}^{1}+\cdots+\mathscr{D}_{+}^{N}, \quad \mathscr{D}_{-}=\mathscr{D}_{-}^{1}+\cdots+\mathscr{D}_{-}^{N} .
$$

We denote by $\mathscr{D}^{\prime}$ the union

$$
\mathscr{D}_{ \pm}^{\prime}=\bigcup U(t) \mathscr{D}_{ \pm} \cdot
$$

LEMMA 1.5. $R_{ \pm}^{F}$ is an isometry on $\mathscr{D}_{ \pm}^{\prime}$.

Proof. Since $U(t)$ is an isometry and $R_{ \pm}^{F}$ are translation representations, it suffices to show that, say, $R_{+}^{F}$ is an isometry on $\mathscr{D}_{+}$. We assume without loss of generality that the $a_{j}$ are so large that the cusp neighborhoods $N_{j}$, defined in (1.26), are disjoint. In this case the linear spaces on the right in (1.30) are orthogonal, and every $f$ in $\mathscr{D}_{+}$can be decomposed as the orthogonal sum

$$
f=\sum_{1}^{N} d^{j}, \quad d^{j} \in \mathscr{D}_{+}^{j} .
$$

Since the $N_{j}$ are disjoint

$$
E_{F}(f)=\sum E_{F}\left(d^{j}\right) .
$$

We shall show that for $d^{j}$ of form (1.28)

$$
R_{+}^{k} d^{j}=\delta_{j k}\left(2\left|F_{j}\right|\right)^{1 / 2} l,
$$

where $\delta_{j k}$ is the Kronecker symbol, and $l$ is the function entering the definition (1.28) of $d_{+}^{j}$.

A straightforward calculation using (1.28) and (1.17) shows that (1.33) holds for $k=j$.

We turn now to the case $k \neq j$. Since $d^{j}$ is supported in $N_{j}$, it vanishes in $N_{k}$ and so from the definition (1.17) of $R_{+}^{k}$ we conclude that for $k \neq j$,

$$
\left(R_{+}^{k} d^{j}\right)^{+}(s)=0 \text { for } s>\log a_{k} \text {. }
$$

It follows from $(1.18)_{+}$and (1.28) that for $t>0, U(t) d^{j}$ is supported in $N_{j}$. Therefore (1.34) holds for $U(t) d^{j}$ in place of $d^{j}$; using (1.18) + this asserts

$$
\left(R_{+}^{k} U(t) d^{j}\right)(s)=\left(R_{+}^{k} d^{j}\right)(s-t)=0 \text { for } s>\log a_{k} .
$$

Since $t$ is an arbitrary positive number, it follows that for $k \neq j, R_{+}^{k} d^{j} \equiv 0$, as asserted in (1.33).

A brief calculation, using the definition (1.3) of $E_{F}$ and the definition (1.27) $)_{+}$of $d^{j}$, combined with (1.33) for the case $k=j$, shows that

$$
E_{F}\left(d^{j}\right)=2|F| \int|l(s)|^{2} d s=\left\|R_{+}^{j} d^{j}\right\|^{2} .
$$

Combining this with $(1.32)^{\prime}$ we deduce that $R_{+}^{F}$ is an isometry; this completes the proof of Lemma 1.5. 
We now define the space $\mathscr{H}_{+}$to be the closure in the energy norm of $Q \mathscr{D}_{+}^{\prime}$, and similarly $\mathscr{H}_{-}$to be the closure of $Q \mathscr{D}_{-}^{\prime}$. It is clear from the definitions (1.30) and (1.31) that $\mathscr{D}_{+}^{\prime}$ is invariant under $U(t)$. Since $U(t)$ commutes with $Q$, by $(1.16)$, it follows that $\mathscr{H}_{+}$is also invariant under the action of $U(t)$, and so is $\mathscr{H}_{-}$.

LEMMA 1.6. $R_{+}^{F}$ is an isometry on $\mathscr{H}_{+}$; likewise $R_{-}^{F}$ is an isometry on $\mathscr{H}_{-}$.

Proof. It suffices to show that $R_{+}^{F}$ is an isometry on $Q \mathscr{D}_{+}^{\prime}$. According to Lemma $1.5, R_{+}^{F}$ is an isometry on $\mathscr{D}_{+}^{\prime}$; therefore Lemma 1.6 would follow from the observation that the projection $Q$ does not alter the $E_{F}$ norm of an element $g$ of $\mathscr{D}_{+}^{\prime}$ nor the value of $R_{+}^{F} g$. I.e., for every $g$ in $\mathscr{D}_{+}^{\prime}$,

$$
E_{F}(Q g)=E_{F}(g)
$$

and

$$
R_{+}^{F} Q g=R_{+}^{F} g
$$

By definition of the projection $Q$,

$$
g=Q g+p_{-}+p_{+},
$$

where $p_{-}$is a linear combination of the $\left\{p_{k}^{-}\right\}$and $p_{+}$is a linear combination of the $\left\{p_{k}^{+}\right\}$defined in (1.10). The key to (1.35) and (1.36) is the following result:

$$
g=Q g+p_{-} \text {for } g \text { in } \mathscr{D}_{+}^{\prime} .
$$

To prove this we make use of the relations (1.11), $(1.11)^{\prime}$; clearly, to show that there are no $p_{+}$components in $(1.37)^{\prime}$, we have to show that for $g$ in $\mathscr{D}_{+}^{\prime}$

$$
E_{F}\left(g, p_{k}^{-}\right)=0 \text {. }
$$

Every $g$ in $\mathscr{D}_{+}^{\prime}$ is of the form $g=U(t) f, f$ in $\mathscr{D}_{+}$. Using this in (1.38), as well as the invariance of $E_{F}$ and the fact that $U(t) p_{k}^{-}=e^{-\lambda_{k} t} p_{k}^{-}$, we get

$$
E_{F}\left(g, p_{k}^{-}\right)=E_{f}\left(U(t) f, p_{k}^{-}\right)=E_{F}\left(f, U(-t) p_{k}^{-}\right)=e^{\lambda_{k} t} E_{F}\left(f, p_{k}^{-}\right) .
$$

Every $f$ in $\mathscr{D}_{+}$can, by definition, be written as a sum of elements $d^{j}$ of $\mathscr{D}_{+}^{j}$ (see (1.32)); therefore, in view of $(1.38)^{\prime}$, in order to prove (1.38) it suffices to show that

$$
E_{F}\left(d^{j}, p_{k}^{-}\right)=0 \text { for } d^{j} \text { in } D_{+}^{j} \text {. }
$$

By definition (1.28), $d^{j}$ is independent of $x$ in $N_{j}$, and zero outside $N_{j}$. Therefore

$$
E_{f}\left(d^{j}, p_{k}^{-}\right)=\left|F_{j}\right|^{1 / 2} E_{F}\left(d^{j}, \overline{p_{k}^{-}}\right),
$$

where the bar denotes the mean value defined in (1.17). We compute now $\overline{p_{k}}$.

By definition (1.10), and dropping the subscript $k, p^{-}=(\varphi,-\lambda \varphi)$. Thus

$$
\overline{p^{-}}=(\bar{\varphi},-\lambda \bar{\varphi}) \text {. }
$$

As noted before, $e^{\lambda t} \varphi$ satisfies the wave equation; thus $e^{\lambda t} \bar{\varphi}$ satisfies the reduced equation (1.19):

$$
\lambda^{2} \bar{\varphi}=y^{2} \bar{\varphi}_{y y}-(n-2) y \bar{\varphi}_{y}+\left(\frac{n-1}{2}\right)^{2} \bar{\varphi}
$$


This equation is satisfied by the powers $y^{(n-1) / 2 \pm \lambda}$; all other solutions are linear combinations of them. Since $\varphi$ is square integrable in $N_{j}$ with respect to $d x d y / y^{n}, \bar{\varphi}$ is $L_{2}(a, \infty)$ with respect to $d y / y^{n}$; thus

$$
\bar{\varphi}=\text { const } y^{(n-1) / 2-\lambda} \text {. }
$$

Setting this into (1.40) gives

$$
\overline{p^{-}}=\text {const }\left(y^{(n-1) / 2-\lambda},-\lambda y^{(n-1) / 2-\lambda}\right) \text {. }
$$

Substituting (1.40)' for $\overline{p_{k}}$ and (1.28) for $d_{+}$into $(1.39)^{\prime}$, and using the definition (1.3) of $E_{F}$ shows, after a brief calculation, that (1.39)' is zero. This proves (1.39), and thereby (1.38). From this (1.37)' follows.

We are now ready to prove (1.35) and (1.36). By (1.37)',

$$
E_{F}(Q g)=E_{F}\left(g-p_{-}\right)=E_{F}(g)-2 E_{F}\left(g, p_{-}\right)+E_{F}\left(p_{-}\right) .
$$

The second term on the right is zero by (1.38), and the third team by (1.11); this proves (1.35).

We turn now to (1.36). By (1.37)',

$$
R_{+}^{F} Q g=R_{+}^{F} g-R_{+}^{F} p_{-} .
$$

We claim that

$$
R_{+}^{F} p_{-}=0 .
$$

To see this we note that, by definition, $p_{-}$is a linear combination of the $p_{k}^{-}$. Setting formula $(1.40)^{\prime}$ for $\overline{p_{-}}$into the definition $(1.17)_{+}$of $R_{+}^{j}$, and using $y=e^{s}$ with $\partial_{s}=y \partial_{y}$, we obtain

$$
R_{+}^{j} p^{-}=\text {const }\left\{y \partial_{y} y^{(1-n) / 2} y^{(n-1) / 2-\lambda}+y^{(1-n) / 2} \lambda y^{(n-1) / 2-\lambda}\right\}=0 .
$$

This proves $(1.42)^{\prime}$; combining $(1.42)^{\prime}$ with (1.42) we obtain (1.36). This completes the proof of Lemma 1.6.

It follows from (1.33) that the range of $R_{+}^{j}$ on $\mathscr{D}_{+}$includes all $C_{0}^{\infty}\left(\log a_{j}, \infty\right)$ functions $l$. Since $R_{+}^{j}$ is a translation representation, it follows that the range of $R_{+}^{j}$ on $\mathscr{D}_{+}^{\prime}$ includes all $C_{0}^{\infty}(\mathbf{R})$ functions. From this and (1.36) it follows that the range of $R_{+}^{F}$ on $Q \mathscr{D}_{+}^{\prime}$ is dense in $L^{2}(\mathbf{R})^{N}$. Combining this with Lemma 1.6 we obtain

THEOREM 1.7. $R_{+}^{F}$ is a unitary translation representation of $\mathscr{H}_{+}$onto $L^{2}(\mathbf{R})^{N}$ and $R_{-}^{F}$ is a unitary anti-translation representation of $\mathscr{H}_{-}$onto $L^{2}(\mathbf{R})^{N}$. For $f$ in $\mathscr{H}_{+}$

$$
R_{+}^{F} U(t) f=T(t) R_{+}^{F} f, \quad E_{F}(f)=\left\|R_{+}^{F} f\right\|^{2} ;
$$

and for $f$ in $\mathscr{H}_{-}$

$$
R_{-}^{F} U(t) f=T(-t) R_{-}^{F} f, \quad E_{F}(f)=\left\|R_{-}^{F} f\right\|^{2} .
$$

Since Fourier transform changes a translation representation into a spectral representation, an immediate corollary of Theorem 1.7 is

COROLlaRY 1.8. $U(t)$ has an absolutely continuous spectrum on $\mathscr{H}_{+}$and on $\mathscr{H}_{-}$. The infinitesimal generator $A$ of $U(t)$ has an absolutely continuous spectrum of uniform multiplicity $N$ on $\mathbf{R}$. 
We shall also require the following results:

Lemma 1.9. If $g$ lies in $\mathscr{H}_{Q}$, then $R_{+}^{F} g \equiv 0$ iff $g$ is orthogonal to $\mathscr{H}_{+}$. Similarly $R_{-}^{F} \equiv 0$ iff $g$ is orthogonal to $\mathscr{H}_{-}$.

Proof. $g$, being in $\mathscr{H}_{Q}$, is orthogonal to $\mathscr{P}$; hence $E_{F}\left(g, Q d_{+}^{\prime}\right)=E_{F}\left(g, d_{+}^{\prime}\right)$ for any $d_{+}^{\prime}$ in $\mathscr{H}_{G}$. For $d_{+}$of the form (1.28), an explicit calculation, using (1.17) and (1.3), gives

$$
E_{F}\left(g, d_{+}\right)=\int R_{+}^{j} g(s) \bar{l}(s) d s
$$

So for $d_{+}^{\prime}=U(t) d_{+}$, using $(1.43)_{+}$and (1.44), we get

$$
\begin{aligned}
E_{F}\left(g, d_{+}^{\prime}\right) & =E_{F}\left(g, U(t) d_{+}\right)=E_{F}\left(U(-t) g, d_{+}\right)=\int\left(R_{+}^{j}(U(-t) g)\right) \bar{l}(s) d s \\
& =\int T(-t)\left(R_{+}^{j} g\right) \bar{l}(s) d s=\int\left(R_{+}^{j} g\right) \bar{l}(s-t) d s .
\end{aligned}
$$

Since the index $j$ is arbitrary and the translates of $l$ are dense, Lemma 1.9 follows.

A consequence of this is

LEMMA 1.10. For any $h$ in $\mathscr{H}_{Q}$

$$
E_{F}(h) \geqslant\left\|R_{-}^{F} h\right\|^{2}
$$

Proof. Decompose $h$ into orthogonal parts

$$
h=f+g, \quad f \text { in } \mathscr{H}_{-} \text {and } g \perp \mathscr{H}_{-} .
$$

By Lemma $1.9, R_{-}^{F} g \equiv 0$ so that

$$
R_{-}^{F} h=R_{-}^{F}
$$

Since $f$ and $g$ are orthogonal by construction,

$$
E_{F}(h)=E_{F}(f)+E_{F}(g) \geqslant E_{F}(f) .
$$

In the last step we used the fact that $E_{F} \geqslant 0$ on $\mathscr{H}_{Q}$. On the other hand, by (1.43),

$$
E_{F}(f)=\left\|R_{-}^{F} f\right\|^{2}=\left\|R_{-}^{F} h\right\|^{2} ;
$$

in the last step we have used (1.47). Combining these last two relations, we obtain (1.45).

We denote by $T$ the time reversal operator mapping $f=\left\{f_{1}, f_{2}\right\}$ into $T f=$ $\left\{f_{1},-f_{2}\right\}$. It is obvious from (1.28) that $T \mathscr{D}_{+}=\mathscr{D}_{-}$, and from this it follows that

$$
T \mathscr{H}_{+}=\mathscr{H}_{-} .
$$

LEMMA 1.11. $\mathscr{Z}=$ null space of $A$.

Proof. By definition

$$
\mathscr{Z}=\left[z \in \mathscr{H}_{Q}, E_{F}(z, f)=0 \text { for all } f \text { in } \mathscr{H}_{Q}\right] .
$$

Clearly $E_{F}(z, g)=0$ for all $g$ orthogonal to $\mathscr{H}_{Q}$ and hence, combining this with (1.49), for all $g$ in $\mathscr{H}_{G}$. In particular, it holds for all $g$ of the form $\left\{0, g_{2}\right\}$ with arbitrary $g_{2}$ in $L_{2}(F)$, so that $z_{2}=0$ for $z=\left\{z_{1}, z_{2}\right\}$. It also holds for all 
$g=\left\{g_{1}, 0\right\}$ with arbitrary $g_{1}$ in $C_{0}^{\infty}(F)$, and hence by (1.3), $H_{F}\left(z_{1}, L g_{1}\right)=0$ for all $g_{1}$ in $C_{0}^{\infty}(F)$. Thus $L z_{1}=0$ in the weak sense and hence $A z=0$. Conversely if $A f=0, f=\left\{f_{1}, f_{2}\right\}$, then $f_{2}=0$ and

$$
E_{F}(f, g)=-H_{F}\left(f_{1}, L g_{1}\right)=0
$$

for all $g$ in $C_{0}^{\infty}(F)$. Since $C_{0}^{\infty}(F)$ data are dense in $\mathscr{H}_{G}$, it follows that $E_{F}(f, g)=0$ for all $g$ in $\mathscr{H}_{G}$ and hence that $f$ belongs to $\mathscr{Z}$.

2. Completeness. With these preliminaries out of the way, we are now ready to establish the completeness of the translation representations. More precisely, we shall show that $\mathscr{H}_{+}=\mathscr{H}_{-}$and that this subspace is the orthogonal complement of the point spectrum of $A$ in $\mathscr{H}_{Q}$.

THEOREM 2.1 .

$$
\mathscr{H}_{+}=\mathscr{H}_{-}(\bmod \mathscr{Z}) .
$$

The proof relies on four lemmas.

Define the space $\mathscr{H}_{c}$ as

$$
\mathscr{H}_{c}=\overline{\mathscr{H}_{-}+\mathscr{H}_{-}},
$$

the bar denoting completion with respect to the energy norm. Clearly $\mathscr{H}_{c}$ is invariant under $U(t)$ along with $\mathscr{H}_{-}$and $\mathscr{H}_{+}$; thus $\mathscr{H}_{c}$ is an invariant subspace of the generator $A$.

LEMMA 2.2. The spectrum of $A$ is absolutely continuous on $\mathscr{H}_{c}$.

Proof. Since $\mathscr{H}_{c}$ is invariant under $A$, if the point or singular spectrum of $A$ were nonempty, then there would be a nonzero element $f$ in $\mathscr{H}_{c}$ whose spectrum is purely point or singular. Such an $f$ is orthogonal to all elements having an absolutely continuous spectrum. In particular, $f$ will be orthogonal to $\mathscr{H}_{-}$and $\mathscr{H}_{+}$by Corollary 1.8 and hence by (2.2) to $\mathscr{H}_{c}$. Thus $f$ is the zero element of $\mathscr{H}_{c}$ or, more precisely, $f$ belongs to $\mathscr{Z}$.

Lemma 2.3. Suppose $f$ belongs to $\mathscr{H}_{c}$; denote by $u(t)$ the solution to the wave equation with initial data $f$. Let $S$ be any compact subset of $F$. Then

$$
\lim _{t \rightarrow \infty} H_{S}\left(u_{t}(t)\right)=0 .
$$

Proof. Since $E_{F} \geqslant 0$ on $\mathscr{H}_{c}$ we can write

$$
H_{S}\left(u_{t}(t)\right) \leqslant H_{F}\left(u_{t}(t)\right) \leqslant E_{F}(u(t))=E_{F}(f) .
$$

It therefore suffices to prove (2.3) for a subset of $\mathscr{H}_{c}$ dense relative to $E_{F} \cdot \mathscr{H}_{c} \cap \mathscr{D}(A)$ will serve as this dense subset; here $\mathscr{D}(A)$ denotes the domain of $A$.

For $g=\left\{g_{1}, g_{2}\right\}$ in $\mathscr{H}_{c} \cap \mathscr{D}(A)$ it follows from (1.3) that

$$
H_{F}\left(g_{2}\right) \leqslant E_{F}(g)
$$

and

$$
D_{F}\left(g_{2}\right)-\left(\frac{n-1}{2}\right)^{2} H_{F}\left(g_{2}\right) \leqslant E_{F}(A g)
$$


It follows directly from (2.5) and (2.5)' that

$$
\sum_{|\alpha| \leqslant 1} H_{S}\left(\partial^{\alpha} g_{2}\right) \leqslant c\left[E_{F}(g)+E_{F}(A g)\right] .
$$

This inequality holds in particular for $u_{t}(t)$ :

$$
\sum_{|\alpha| \leqslant 1} H_{S}\left(\partial^{\alpha} u_{t}(t)\right) \leqslant c\left[E_{F}(f)+E_{F}(A f)\right] .
$$

The spectral resolution of $A$ gives the following representation for $U(t)$ :

$$
U(t)=\int e^{i \lambda t} d P(\lambda) .
$$

In particular for any $f, g$ in $\mathscr{H}_{c}$ we have

$$
E_{F}(U(t) f, g)=\int e^{i \lambda t} d E_{F}(P(\lambda) f, g) .
$$

Since $A$ has absolutely continuous spectrum over $\mathscr{H}_{c}$, the measure on the right in (2.7) is absolutely continuous. It therefore follows by the Riemann-Lebesgue lemma that

$$
\lim _{t \rightarrow 0} E_{F}(U(t) f, g)=0
$$

for all $g$ in $\mathscr{H}_{c}$. Since $U(t) f$ belongs to $\mathscr{H}_{c}$ we can replace $g$ in $(2.7)^{\prime}$ by any element in $\mathscr{H}_{G}$. In particular (2.7) holds for all $g$ of the form $\left\{0, g_{2}\right\}$ with $g_{2}$ any element of $L_{2}(F)$ so that

$$
\lim _{t \rightarrow \infty}\left(u_{t}(t), g_{2}\right)=0 \quad \text { for all } g_{2} \in L_{2}(F) .
$$

We see from (2.6) ${ }^{\prime}$ and the Rellich compactness criterion that the $u_{t}(t)$ are compact in the $L^{2}(S)$ norm. According to $(2.7)^{\prime \prime}$, the $u_{t}(t)$ converge weakly to zero as $t \rightarrow \infty$ in $L^{2}(F)$; this combined with compactness in $L^{2}(S)$ shows that the $u_{t}(t)$ converge strongly to zero in the $L^{2}(S)$ norm. This completes the proof of Lemma 2.3.

LEMMA 2.4. If $f$ is in $\mathscr{H}_{c}$ and $R_{+}^{F} f=0$, then

$$
\lim _{t \rightarrow \infty} H_{F}\left(u_{t}(t)\right)=0 \text {. }
$$

Proof. We saw earlier that if $R_{+}^{F} f=0$, then $R_{+}^{F} U(t) f=0$ for all $t$; it follows then also that

$$
R_{+}^{F} f_{\varphi}=0
$$

where

$$
f_{\varphi}=\int \varphi(t) U(t) f d t .
$$

As $\varphi$ tends to the $\delta$ function, $f_{\varphi}$ tends to $f$ in the $E_{F}$ norm. Now for $f$ in $\mathscr{H}_{c}$

$$
H_{F}\left(u_{t}(t)\right) \leqslant E_{F}(u(t) f)=E_{F}(f) .
$$

Hence it follows that if (2.8) holds for all $f_{\varphi}$, it also holds for $f$. Since $f$ belongs to the domain of $A$ for $\varphi$ in $C_{0}^{1}$, it follows that it suffices to prove Lemma 2.4 for $f$ in the domain of $A$. 
For $f$ in $\mathscr{D}(A)$ both $U(t) f$ and $A U(t) f$ lie in $\mathscr{H}_{G}$ and are of finite energy. Since energy is conserved, it follows from (1.3) that both $H_{F}\left(u_{t}(t)\right)$ and $D_{F}\left(u_{t}(t)\right)$ are uniformly bounded:

$$
H_{F}\left(u_{t}(t)\right)+D_{F}\left(u_{t}(t)\right) \leqslant \text { const. }
$$

Next let $\left\{\psi_{k}\right\}$ be the normalized eigenfunctions of $-\Delta_{x}$, the negative of the Euclidean Laplace operator, for $\Gamma_{j}$-automorphic functions over $F_{j}$. Denote the corresponding eigenvalues by $\left\{\omega_{k}\right\}$. Since $F_{j}$ is a compact domain, $\Delta_{x}$ has a standard discrete spectrum. The lowest eigenvalue $\omega_{0}$ is zero with multiplicity one, and the corresponding eigenfunction $\psi_{0}$ is constant:

$$
\psi_{0}=\left|F_{j}\right|^{-1 / 2}, \quad \psi_{1}, \ldots
$$

and

$$
0=\omega_{0}<\omega_{1} \leqslant \cdots .
$$

Setting $g=u_{t}(t)$ for fixed $t$, we expand $g(x, y)$ as a function of $x$ into a Fourier series with respect to these eigenfunctions:

$$
g(x, y)=\sum_{0}^{\infty} g^{(k)}(y) \psi_{k}(x) .
$$

We have the two Parseval relations

$$
\int_{F_{j}}|g|^{2} d x=\sum_{0}^{\infty}\left|g^{(k)}\right|^{2}
$$

and

$$
\int_{F_{j}}\left|g_{x}\right|^{2} d x=-\int_{F_{j}} g \overline{\Delta g} d x=\sum_{1}^{\infty} \omega_{k}\left|g^{(k)}\right|^{2}
$$

Integrating (2.14) with respect to $d y / y^{n}$ from $a$ to $\infty$ and (2.15) with respect to $d y / y^{n-2}$ gives

$$
H_{N_{j}}(g)=\int_{N_{j}}|g|^{2} \frac{d x d y}{y^{n}}=\sum_{0}^{\infty} \int_{a}^{\infty}\left|g^{(k)}(y)\right|^{2} \frac{d y}{y^{n}}
$$

and

$$
\int_{N_{j}}\left|g_{x}\right|^{2} \frac{d x d y}{y^{n-2}}=\sum_{1}^{\infty} \omega_{k} \int_{a}^{\infty}\left|g^{(k)}(y)\right|^{2} \frac{d y}{y^{n-2}}
$$

where again

$$
N_{j}=F_{j} x(a, \infty)
$$

The left side of $(2.15)^{\prime}$ is $\leqslant D_{N_{j}}(g)$. Thus

$$
D_{N_{j}}(g) \geqslant \sum_{1}^{\infty} \omega_{k} \int_{a}^{\infty} y^{2}\left|g^{(k)}(y)\right|^{2} \frac{d y}{y^{n}} \geqslant a^{2} \omega_{1} \sum_{1}^{\infty} \int_{a}^{\infty}\left|g^{(k)}\right|^{2} \frac{d y}{y^{n}} .
$$


Combining (2.17) with $(2.14)^{\prime}$ we obtain

$$
H_{N_{j}}(g) \leqslant \int_{a}^{\infty}\left|g^{(0)}(y)\right|^{2} \frac{d y}{y^{n}}+\frac{1}{a^{2} \omega_{1}} D_{N_{j}}(g) \text {. }
$$

We obtain an estimate for $g^{(0)}$ by making use of the relation $g^{(0)}=\bar{u}_{t}(t)$. Using the definition (1.17) of $R_{+}^{j}$, we see that if $R_{+}^{j} f \equiv 0$, then

$$
\partial_{s} e^{(1-n) s / 2} \overline{f_{1}}=e^{(1-n) s / 2} \overline{f_{2}} .
$$

Using the analogous definition of $R_{-}^{j}$ we conclude from this that

$$
k_{-}(s) \equiv R^{j} f(s)=2 e^{(1-n) s / 2} \overline{f_{2}}(s),
$$

where $k_{-}$abbreviates $R_{-}^{j} f$. Since $R_{+}^{F}$ transmutes $U(t)$ into translation (Lemma 1.4), it follows from the hypothesis $R_{+}^{j} f=0$ that $R_{+}^{j} U(t) f \equiv 0$. Consequently (2.19) holds with $f$ replaced by $U(t) f$. Further, since $R_{-}^{F}$ is an anti-translation representation, we get

$$
k_{-}(s+t)=2 e^{(1-n) s / 2} \overline{u_{t}(t)} \text {. }
$$

Next we define

$$
h(t)=\left\{0, u_{t}(t)\right\} .
$$

Using the definition (1.17) of $R_{-}^{j}$, we get $R_{-}^{j} h(t)=e^{(1-n) s / 2} \overline{u_{t}(t)}$. Combining this with $(2.19)^{\prime}$ we have

$$
k_{-}(s+t)=2 R_{-}^{j} h(t)(s)
$$

It follows by (2.19)' that for all $t$

$$
\begin{aligned}
\int_{a}^{\infty}\left|\bar{u}_{t}(y, t)\right|^{2} \frac{d y}{y^{n}} & =\int_{\log a}^{\infty}\left|e^{(1-n) s / 2} \bar{u}_{t}\left(e^{s}, t\right)\right|^{2} d s \\
& =\frac{1}{4} \int_{\log a}^{\infty}\left|k_{-}(s+t)\right|^{2} d s
\end{aligned}
$$

It follows from Lemma 1.10 that $k_{-}$is square integrable over $R$; therefore it follows from (2.22) that for any $a$ fixed,

$$
\lim _{t \rightarrow \infty} \int_{a}^{\infty}\left|\bar{u}_{t}(y, t)\right|^{2} \frac{d y}{y^{n}}=0
$$

Hence choosing $a$ sufficiently large and combining (2.23) with (2.18) we get

$$
\lim _{t \rightarrow \infty} H_{N_{j}}\left(u_{t}(t)\right) \leqslant \mathrm{const} / a^{2} \text {. }
$$

We decompose $F$ as $F=\cup N_{j} \cup S, S$ compact, and write

$$
H_{F}\left(u_{t}\right)=\sum_{1}^{N} H_{N_{j}}\left(u_{t}\right)+H_{S}\left(u_{t}\right) .
$$

Applying (2.3) and (2.24) to the right member of (2.25), we conclude,

$$
\lim _{t \rightarrow \infty} H_{F}\left(u_{t}(t)\right) \leqslant \text { const } / a^{2} \text {. }
$$

Since $a$ can be chosen arbitrarily large, (2.8) follows. This concludes the proof of Lemma 2.4 . 
LeMMA 2.5. If $f$ is in $\mathscr{H}_{c}$ and $R_{+}^{F} f=0$, then $f$ belongs to $\mathscr{Z}$.

Proof. We may as well assume that $f$ belongs to $\mathscr{H}_{c} \cap \mathscr{D}\left(A^{2}\right)$ since any $f$ in $\mathscr{H}_{c}$ can be approximated by data of the form $f_{\varphi}$, as in (2.10), which belongs to $\mathscr{H}_{c} \cap \mathscr{D}\left(A^{2}\right)$. For such data we again have $R_{+}^{F} f_{\varphi}=0$. We shall prove that $f_{\varphi}$ lies in $\mathscr{Z}$. As $\varphi$ tends to the $\delta$-function, $f_{\varphi}$ tends to $f$; and since $\mathscr{Z}$ is closed, $f$ itself will belong to $\mathscr{Z}$.

Since $R_{+}^{F} A^{j} f=\left(-\partial_{s}\right)^{j} R_{+}^{F} f=0$ for $j=0,1,2$, it follows from Lemma 2.4, applied to the second components of $A^{j} U(t) f$, that

$$
\lim _{t \rightarrow \infty} H_{F}\left(u_{t}(t)\right)=0, \quad \lim _{t \rightarrow \infty} H_{F}(L u(t))=0, \quad \lim _{t \rightarrow \infty} H_{F}\left(L u_{t}(t)\right)=0 .
$$

For $v=u_{t}$, we have $v_{t}=u_{t}=L u$ and $L v=L u_{t}$. By (1.3), $E_{F}(v)=-H_{F}(v, L v)+$ $H_{F}\left(v_{t}\right)$. It follows therefore from (2.26) that $\lim _{t \rightarrow \infty} E_{F}(v(t))=0$. Since $E_{F}$ is invariant in $t$, we get

$$
E_{F}(A f)=E_{F}(v(0))=\lim _{t \rightarrow \infty} E_{F}(v(t))=0 .
$$

Consequently $A f=0 \bmod \mathscr{Z}$. Now $f$ belongs to $\mathscr{H}_{c}$ and by Lemma $2.2, A$ is absolutely continuous on $\mathscr{H}_{c}$; it follows that if $f$ is a null vector of $A(\bmod \mathscr{Z})$, then $f$ lies in $\mathscr{Z}$. This proves Lemma 2.5 .

Theorem 2.1 is an immediate consequence of Lemma 2.5. For if $\mathscr{H}_{+} \neq \mathscr{H}_{-} \bmod \mathscr{Z}$, then $\mathscr{H}_{+}$, say, would be a proper subspace of $\mathscr{H}_{c}$ and there would be a nonzero $f$ in $\mathscr{H}_{\text {c }}$ orthogonal to $\mathscr{H}_{+}$. According to Lemma 1.9 , for such an $f$ we would have $R_{+}^{F} f=0$. According to Lemma 2.5, $f$ lies in $\mathscr{Z}$. This completes the proof of Theorem 2.1.

We go back now to inequality (2.18) and note that it has the following immediate corollary:

LEMMA 2.6. Suppose that the automorphic functions $g$ have finite square and Dirichlet integrals over $F$ and that $\bar{g}=0$ in the jth cusp. Then given any $\varepsilon>0$, we can choose a neighborhood $N_{j}$ of the type (2.16) with a so large that for all such functions

$$
H_{N_{j}}(g)=\int_{N_{j}}|g|^{2} \frac{d x d y}{y^{n}}<\varepsilon D_{F}(g)
$$

In what follows we denote $H_{+}=H_{-}$by $H_{c}$. The second main result of this section is

THEOREM 2.7. Denote by $\mathscr{H}_{p}$ the orthogonal complement of $\mathscr{H}_{c}$ in $\mathscr{H}_{Q}$ :

$$
\mathscr{H}_{p}=\mathscr{H}_{Q} \ominus \mathscr{H}_{c} .
$$

$\mathscr{H}_{p}$ is an invariant subspace of $U(t)$ and $A$ has a standard pure point spectrum over $\mathscr{H}_{p}$; i.e. each eigenvalue has finite multiplicity and the eigenvalues have no finite point of accumulation.

Remark. As before, the assertion of Theorem 2.7 must be taken modulo $\mathscr{Z}$. According to Lemma $1.11, \mathscr{Z}=$ null space of $A$; i.e. $\mathscr{Z}$ consists of all data of the form $\{v, 0\}$ in $\mathscr{H}_{G}$ with $L v=0$ where $v$ need not be square integrable. Thus if $A f=\lambda f$ modulo $\mathscr{Z}$, then

$$
f_{2}=\lambda f_{1}+v \text { and } L f_{1}=\lambda f_{2}
$$


If $\lambda \neq 0$, then setting $\chi=f_{1}+v / \lambda$, we see that $f_{2}=\lambda \chi, L \chi=\lambda^{2} \chi$ and $A\{\chi, \lambda \chi\}$ $=\lambda\left\{\chi, \lambda_{\chi}\right\}$; that is, $\{\chi, \lambda \chi\}$ is a genuine eigenvector of $A$. However if $\lambda=0, f$ may be of the form $\{v, \chi\}$, where $\chi \in L_{2}(F)$ and $L \chi=0$. In this case $A\{v, \chi\}=$ $\{\chi, 0\}$ need not vanish but does lie in $\mathscr{Z}$.

Proof. Both $\mathscr{H}_{Q}$ and $\mathscr{H}_{c}$ are invariant under $U(t)$ and since $U(t)$ is unitary on $\mathscr{H}_{Q}$ it follows that the orthogonal complement $\mathscr{H}_{p}$ is also invariant under $U(t)$. It also follows that $\mathscr{H}_{p}$ is invariant under $(k-A)^{-1}$. Similar reasoning shows that $\mathscr{H}_{p}$ is invariant under the time reversal operator $T$. Thus if $\left\{g_{1}, g_{2}\right\}$ belongs to $\mathscr{H}_{p}$, then so does $\left\{g_{1},-g_{2}\right\}$, and from this it follows that $\left\{0, g_{2}\right\}$ also belongs to $\mathscr{H}_{p}$.

Definition. We denote by $\mathscr{C}$ the space of functions $g$ for which $\{0, g\}$ belongs to $\mathscr{H}_{p} . \mathscr{H}_{p}$ being an orthogonal complement, is closed; and it follows that $\mathscr{C}$ is a closed subspace of $L_{2}(F)$.

LeMmA 2.8. $\left(L-k^{2}\right)^{-1}$ maps $\mathscr{C}$ into $\mathscr{C}$ and is a compact operator on $\mathscr{C}$ for $k$ real $\neq 0$.

Proof. Since $(k-A)^{-1}$ maps $\mathscr{H}_{p}$ into itself, so does $(k-A)^{-1}+(k+A)^{-1}=$ $2 k\left(k^{2}-A^{2}\right)^{-1}$. Now

$$
A=\left(\begin{array}{cc}
0 & I \\
L & 0
\end{array}\right) \text { and } A^{2}=\left(\begin{array}{cc}
L & 0 \\
0 & L
\end{array}\right)
$$

So $\left(k^{2}-A^{2}\right)^{-1}$ acts on each component $\left(k^{2}-L\right)^{-1}$. Applying this to elements of $\mathscr{H}_{p}$ of the form $\{0, g\}$, we conclude that $\left(k^{2}-L\right)^{-1}$ maps $\mathscr{C}$ into $\mathscr{C}$, boundedly.

To establish compactness, we note that by definition every element $f$ of $\mathscr{H}_{p}$ is orthogonal to $\mathscr{H}_{+}$. According to Lemma 1.9, this implies that $R_{+}^{F} f=0$. Taking $f$ to be of the form $\{0, g\}, g$ in $\mathscr{C}$, we conclude, using the definition (1.17) of the components of $R_{+}^{F}$, that for $g$ in $\mathscr{C}$

$$
\bar{g}=0 \text { in every cusp. }
$$

Next let $h$ be any function in $\mathscr{C}$ of $L_{2}$ norm $\leqslant 1$ :

$$
H_{F}(h) \leqslant 1 \text {, }
$$

and let $g$ be its image under $\left(k^{2}-L\right)^{-1}$ :

$$
g=\left(k^{2}-L\right)^{-1} h
$$

so that

$$
k^{2} g-L g=h .
$$

Taking the scalar product with $g$ we get, using (1.2),

$$
\left(k^{2}-\left(\frac{n-1}{2}\right)^{2}\right) H_{F}(g)+D_{F}(g)=H_{F}(h, g)
$$

by the Schwarz inequality,

$$
\left|H_{F}(h, g)\right| \leqslant \frac{1}{2} H_{F}(h)+\frac{1}{2} H_{F}(g) .
$$

Hence choosing $k$ so large that $k^{2}-((n-1) / 2)^{2}>\frac{3}{2}$, we obtain, using (2.29), that

$$
H_{F}(g)+D_{F}(g) \leqslant \frac{1}{2} \text {. }
$$


Since $\left(k^{2}-L\right)^{-1}$ maps $\mathscr{C}$ into $\mathscr{C}, g$ belongs to $\mathscr{C}$ and hence, by (2.28), $\bar{g}=0$ in every cusp. Therefore Lemma 2.6 is applicable and the inequality (2.27) holds in every cusp. The inequality (2.31) shows that, given any $\varepsilon$, the same neighborhoods $N_{j}$ may be chosen for all $g$ of the form (2.30), provided $h$ belongs to $\mathscr{C}$ and $H_{F}(h) \leqslant 1$.

The complement of the cusps, $S=F \backslash \cup N_{j}$, is a compact set. Therefore by (2.31) and the Rellich compactness theorem, every sequence of $g_{n}$ 's of the form (2.30) has a subsequences which converges in the norm $H_{S}$. Taking a sequence $\varepsilon_{n} \rightarrow 0$ and applying the diagonal process, we can select a subsequence that converges in the $H_{F}$ norm. This completes the proof of Lemma 2.8 .

Since $E_{F}$ and hence $-L$ is nonnegative on $\mathscr{C}$, it follows from Lemma 2.8 that $L$ has a complete set of eigenfunctions $\left\{\chi_{n}\right\}$ in $\mathscr{C}$ with nonpositive eigenvalues $\left\{-\kappa_{n}^{2}\right\}$;

$$
L \chi_{n}=-\kappa_{n}^{2} \chi_{n} \text {. }
$$

Since $\chi_{n}$ is a square integrable eigenfunction of $L$ it also has a finite Dirichlet integral and is orthogonal to the eigenfunction $\varphi_{j}$ in (1.9). Therefore $\left\{\chi_{n}, 0\right\}$ belongs to $\mathscr{H}_{Q}$. We use these eigenpairs to construct eigendata for $A$. Set

$$
\begin{aligned}
& q_{n}^{ \pm}=\left\{\chi_{n}, \pm \kappa_{n} \chi_{n}\right\} \quad \text { for } \kappa_{n} \neq 0, \\
& \theta_{n}=\left\{0, \chi_{n}\right\}, \quad \nu_{n}=\left\{\chi_{n}, 0\right\} \text { for } \kappa_{n}=0 .
\end{aligned}
$$

Then

$$
\begin{aligned}
& A q_{n}^{ \pm}= \pm \kappa_{n} q_{n}^{ \pm}, \quad A \nu_{n}=0, \\
& A \theta_{n}=\nu_{n} \text { and } A^{2} \theta_{n}=0 .
\end{aligned}
$$

We now define $\mathscr{H}_{p}^{\prime}$ to be the closure of the subspace spanned by the data (2.33). It obviously belongs to the point spectrum of $A$ and hence is orthogonal to $\mathscr{H}_{c}$, which by Corollary 1.8 , has an absolutely continuous spectrum; so by $(2.27), \mathscr{H}_{p}^{\prime} \subset \mathscr{H}_{p}$. It is clear from (2.34) that $\mathscr{H}_{p}^{\prime}$ is an invariant subspace for $U(t)$ and therefore so is its orthogonal complement in $\mathscr{H}_{p}$ :

$$
\mathscr{Z}^{\prime}=\mathscr{H}_{p} \ominus \mathscr{H}_{p}^{\prime}
$$

Since the $\left\{\chi_{n}\right\}$ form a complete set of eigenfunctions in $C$ and the second component of any vector in $\mathscr{Z}^{\prime}$ belongs to $C$, we see that the second component of any $f$ in $\mathscr{Z}^{\prime}$ vanishes.

Suppose now that $f$ belongs to $\mathscr{Z}^{\prime}$. We can approximate $f$ in $\mathscr{Z}^{\prime}$ by an element $f_{\varphi}$ of the form (2.10) in the domain of $A$; we denote $f_{\varphi}$ by $g=\left\{g_{1}, g_{2}\right\}$. Since $\mathscr{Z}^{\prime}$ is an invariant subspace, both $g$ and $A g$ lie in $\mathscr{Z}^{\prime}$, and, as noted above, have second components equal to 0 ; i.e. $g_{2}=0$ and $L g_{1}=0$. Then $g$ lies in $\mathscr{Z}$ by Lemma 1.11 . Since such data are dense in the $G$ norm in $\mathscr{Z}^{\prime}$, we may conclude that $\mathscr{Z}^{\prime} \subset \mathscr{Z}$ and hence that $\mathscr{H}_{p}^{\prime}=\mathscr{H}_{p}$, modulo $\mathscr{Z}$. To complete the proof of Theorem 2.7, we have only to note that the finite dimensionality of $\mathscr{Z}$ was established in Lemma 1.3.

\section{A spectral representation for $L$. As a corollary to Theorem 2.7 we obtain}

THEOREM 3.1. The operator $L$ acting on $L_{2}(F)$ has a standard discrete spectrum plus an absolutely continuous spectrum of uniform multiplicity $N$ on $(-\infty, 0]$.

Proof. Since $\mathscr{P}, \mathscr{H}_{p}$ and $\mathscr{H}_{c}$ are all invariant under the time reversal operator $T$, it follows that the second component of data in $\mathscr{H}_{G}$, which fill out $L_{2}(F)$, can be 
decomposed into point eigenfunctions of $L$ (that is the $\varphi$ 's of (1.9) and the $\chi$ 's of (2.32)) and

$$
\mathscr{F}_{2}=\left[g \text { in } \mathscr{H}_{c} \text { of the form }\left\{0, g_{2}\right\}\right] .
$$

According to Lemma 2.2, the spectrum of $L$ in $\mathscr{F}_{2}$, which is the same as that of $A^{2}$ in $\mathscr{F}_{2}$, is absolutely continuous. We need only to prove that it is of uniform multiplicity $N$ on $(-\infty, 0]$.

To this end we decompose $\mathscr{H}_{c}$ into two orthogonal parts: $\mathscr{F}_{2}$ and

$$
\mathscr{F}_{1}=\left[g \text { in } \mathscr{H}_{c} \text { of the form }\left\{g_{1}, 0\right\}\right] \text {. }
$$

As remarked after Theorem 1.7, a spectral representation of $A$ in $\mathscr{H}_{c}$ is obtained by Fourier transforming $R_{+}^{F}$ :

$$
\tilde{f}(\sigma)=\frac{1}{\sqrt{2 \pi}} \int_{-\infty}^{\infty} e^{i \sigma s} R_{+}^{F} f(s) d s .
$$

Under this map the actions of $A$ and $A^{2}$ go into multiplication by $i \sigma$ and $-\sigma^{2}$, respectively. Since $A^{2}$ acts as $L$ on each component we obtain in this way a spectral representation for $L$ on $\mathscr{F}_{2}$. We denote the Fourier image of $\mathscr{F}_{i}$ by $\tilde{\mathscr{F}}_{i}, i=1,2$. It follows from Theorem 2.1 and Corollary 1.8 via the Plancherel theorem that

$$
\tilde{\mathscr{F}}_{1} \oplus \tilde{\mathscr{F}}_{2}=L_{2}(\mathbf{R})^{N} \text {. }
$$

It is clear from the expression (1.14) for $A$ that $A \mathscr{F}_{1} \subset \mathscr{F}_{2}$ and $A \mathscr{F}_{2} \subset \mathscr{F}_{1}$, whenever this is meaningful. Likewise $\left(k^{2}-A^{2}\right)^{-1} \mathscr{F}_{i} \subset \mathscr{F}_{i}$ for $i=1,2$.

As a consequence

$$
A\left(k^{2}-A^{2}\right)^{-1} \mathscr{F}_{1} \subset \mathscr{F}_{2} \text { and } A\left(k^{2}-A^{2}\right)^{-1} \mathscr{F}_{2} \subset \mathscr{F}_{1} .
$$

The spectral representator of $A\left(k^{2}-A^{2}\right)^{-1}$ is multiplication by $i \sigma /\left(k^{2}+\sigma^{2}\right)$; so it follows that

$$
\frac{\sigma}{k^{2}+\sigma^{2}} \tilde{\mathscr{F}}_{1} \subset \tilde{\mathscr{F}}_{2} \text { and } \frac{\sigma}{k^{2}+\sigma^{2}} \tilde{\mathscr{F}}_{2} \subset \tilde{\mathscr{F}}_{1} .
$$

This result can be strengthened.

LeMma 3.2. Let $J$ denote multiplication by $\operatorname{sgnm}(\sigma)$ :

$$
J \tilde{f}(\sigma)= \begin{cases}\tilde{f}(\boldsymbol{\sigma}) & \text { for } \boldsymbol{\sigma}>0 \\ -\tilde{f}(\boldsymbol{\sigma}) & \text { for } \boldsymbol{\sigma}<0 .\end{cases}
$$

$J$ is a unitary map taking $\tilde{F}_{1}$ onto $\tilde{F}_{2}$ and $\tilde{F}_{2}$ onto $\tilde{F}_{1}$.

Before proving this lemma, we show how it can be used to complete the proof of Theorem 3.1. For $\tilde{f}$ in $\tilde{\mathscr{F}}_{2}, J \tilde{f}$ lies in $\tilde{\mathscr{F}}_{1}$ and is therefore orthogonal to $\tilde{f}$. The relation $(\tilde{f}, J \tilde{f})=0$ can be rewritten as

$$
\int_{0}^{\infty}|\tilde{f}|^{2} d \sigma-\int_{-\infty}^{0}|\tilde{f}|^{2} d \sigma=0
$$

Denoting the restriction map of $L_{2}(\mathbf{R})^{N}$ onto $L_{2}\left(\mathbf{R}_{+}\right)^{N}$ by $\tau$,

$$
\tau: \tilde{f} \rightarrow \sqrt{2} \tilde{f}(\sigma) \text { for } \sigma>0,
$$


it follows from (3.6) that $\tau$ maps $\tilde{\mathscr{F}}_{2}$ isometrically into $L_{2}\left(\mathbf{R}_{+}\right)^{N}$. It remains only to show that this map is onto $L_{2}\left(\mathbf{R}_{+}\right)^{N}$. Since $\tau$ is an isometry, its range is closed and hence if it is not onto there exists a nonzero element $\tilde{h}$ in $L_{2}\left(\mathbf{R}_{+}\right)^{N}$ orthogonal to $\tau \tilde{F}_{2}$. Extend $\tilde{h}$ to be zero for $\sigma<0$. Then for the extended $\tilde{h},(\tilde{f}, \tilde{h})=(\tau \tilde{f}, \tau \tilde{h})=0$ for all $\tilde{f}$ in $\mathscr{F}_{2}$. Thus $\tilde{h}$ lies in $\tilde{F}_{1}$. However since $\tilde{h}(\sigma)$ vanishes on $\mathbf{R}_{-}, \tilde{h}=J \tilde{h}$ and by the lemma, $J \tilde{h}$ belongs to $\tilde{F}_{2}$. Thus $\tilde{h}$ belongs to two orthogonal sets and is therefore zero. This contradiction proves that $\tilde{F}_{2}=L_{2}\left(\mathbf{R}_{+}\right)^{N}$ and hence proves the theorem.

Proof of Lemma 3.2. As $k$ varies the set of functions $\sigma /\left(k^{2}+\sigma^{2}\right)$ separates points on $\mathbf{R}$. It follows from the Stone-Weierstrass theorem that odd polynomials in these functions are dense in the sup norm in the set of all odd continuous functions which vanish at $\infty$ : such polynomial multipliers also have the property $(3.4)^{\prime}$. The strong and uniform operator limits of such polynomials continue to have this property. In particular it will be true for

$$
J_{n}= \begin{cases}\operatorname{sgn} \sigma & \text { for } 1 / n<|\sigma|<n \\ 0 & \text { for }|\sigma|>n+1 / n \\ \text { linear } & \text { in between }\end{cases}
$$

Finally $J_{n} \rightarrow J$ in the strong operator topology so it also holds for $J$. Since $J$ is obviously unitary the rest of the assertion of the lemma is immediate.

In $\$ 6$ of [6] we have shown how to express in terms of Eisenstein series the spectral representation obtained from the translation representation.

4. Perturbations with compact support. The above development is easily adapted to handle automorphic solutions of a perturbed wave equation when the support of the perturbation is compact in $F$. In place of the usual hyperbolic metric in $\mathbf{H}^{n}$, we take

$$
d s^{2}=g_{i j} d x_{i} d x_{j}
$$

here $\left(g_{i j}\right)$ is positive definite, $\Gamma$-automorphic, of class $C^{(1)}$ and on $F$ is equal to $\delta_{i j} / x_{n}^{2}$ outside of a compact subset $S$ of the type (1.6). Note that we have replaced $y$ by $x_{n}$.

In place of the operator (1.2) we now treat

$$
L=g^{-1} \partial_{x_{i}} g^{i j} g \partial_{x_{j}}+\left(\frac{n-1}{2}\right)^{2}-q
$$

where $g$ denotes the square root of $\operatorname{det}\left(g_{i j}\right)$ and $q$ is $L_{\infty}, \Gamma$-automorphic and on $F$ vanishes outside of $S$. With a little care one could allow $q$ to be of class $L_{p}(F)$, where $p=n / 2$ for $n>4, p>2$ for $n=4$ and $p=2$ for $n<4$. However to simplify the exposition we take $q$ in $L_{\infty}$.

The $L_{2}$ and Dirichlet integrals are now of the form

$$
H_{F}(u)=\int_{F}|u|^{2} g d x \text { and } \quad D_{F}(u)=\int_{F} g^{i j} \partial_{x_{i}} u \overline{\partial_{x_{j}} u} g d x
$$

The perturbed wave equation is

$$
u_{t}=L u
$$


and the associated energy form is

$$
\begin{aligned}
E_{F}(u) & =-H_{F}(u, L u)+H_{F}\left(u_{t}\right) \\
& =D_{F}(u)+\int_{F} q|u|^{2} g d x-\left(\frac{n-1}{2}\right)^{2} H_{F}(u)+H_{F}\left(u_{t}\right) .
\end{aligned}
$$

Notice that in the cusp neighborhoods both $L$ and $E_{F}$ are equal to their unperturbed counterparts.

Again defining $K$ as in (1.4), we prove

LEMMA 4.1. The form

$$
G=E_{F}+K
$$

is positive definite provided the constant $c$ and the compact set $S$ are sufficiently large.

The proof of this assertion is essentially the same as that of Lemma 1.1; the same integration by parts in the cusp neighborhoods is used. Further since $q$ is bounded, the extra term $\int q|u|^{2} g d x$ is obviously majorized by $K$.

We again complete the set of $C_{0}^{\infty}(F)$ automorphic data with respect to the $G$-norm and denote the resulting Hilbert space by $\mathscr{H}_{G}$. We note that for data $f$ in $\mathscr{H}_{G}$, the local $L_{2}$ and Dirichlet norms of $f_{1}$ are majorized by $G(f)$.

We then proceed more-or-less as before. The principal difference occurs in the definition of the incoming and outgoing translation representations. To begin with we define $R^{j}{ }_{ \pm}$by (1.17) only for $s>a_{j}$. The argument used in the proof of Lemma 1.4 now shows that

$$
\begin{array}{ll}
R_{+}^{j} U(t) f=T(t) R_{+}^{j} f \quad \text { for } s>\max \left(a_{j}+t, a_{j}\right), \\
R_{-}^{j} U(t) f=T(-t) R_{-}^{j} f \quad \text { for } s>\max \left(a_{j}-t, a_{j}\right) .
\end{array}
$$

We obtain the assertion of Lemma 1.4 over the entire range of $s$ essentially by fiat; that is we define

$$
\begin{array}{ll}
R_{+}^{j} f=T(-t) R_{+}^{j}(U(t) f) & \text { for } s>a_{j}-t, \\
R_{-}^{j} f=T(t) R_{-}^{j}(U(t) f) & \text { for } s>a_{j}+t .
\end{array}
$$

Using the relations (4.7), it is easy to see that $R^{j}{ }_{ \pm} f$ is well defined by (4.8) and satisfies (1.18) for all $s$.

The subspaces $\mathscr{D}_{ \pm}^{j}, \mathscr{D}_{ \pm}$and $\mathscr{D}_{ \pm}^{\prime}$ are defined as before by the relations (1.27)-(1.31). The remaining material in $\$ 1$ carries over verbatim since in all of these proofs the definition (1.17) of $R_{ \pm}^{j} f$ is used only for $s>a_{j_{F}}$.

The proof of completeness for $R_{ \pm}$is basically the same as in $\$ 2$. Since $E_{f}$ now contains the term $\int q|u|^{2} g d x$, the relation $(2.5)^{\prime}$ must be suitably modified. Nevertheless (2.6) continues to hold. Again in the proof of Lemma 2.4, the displayed relations hold only for $s>a_{j}$. However since we are only interested in sufficiently large values of $a$ in (2.22) and (2.23), this restriction is of no consequence. Otherwise all of the material in $\$ \S 2$ and 3 carries over.

We have learned from Peter Sarnak that Peter Perry has also shown that local perturbations of the metric can create no singular spectrum. Perry employs the "geometric" methods of Schrödinger scattering theory. 
In [9], Colin de Verdiere has shown how to construct local, conformal perturbations of the metric in $F$ so that the operator $L$ in (4.2), with $q \equiv 0$, has no negative point spectrum. It follows from our Theorem 3.1 that for such $L, \mathscr{H}_{p}^{\prime}=\{0\}$, so that $\mathscr{H}_{c}=\mathscr{H}_{Q}$. It then follows from Lemma 2.3 that if the initial data $f$ of a solution $u$ of the wave equation are orthogonal to the positive eigenfunctions $\varphi_{j}, j=1, \ldots, m$, then $H_{S}\left(u_{t}(t)\right)$ tends to zero as $t \rightarrow \infty$ for any compact set $S$.

\section{REFERENCES}

1. L. D. Faddeev, Expansion in eigenfunctions of the Laplace operator in the fundamental domain of $a$ discrete group on the Lobačevskii plane, Trudy Moscow. Math. Obšč. 17 (1967), 323-350; English transl., Trans. Moscow Math. Soc. 17 (1967), 357-386.

2. L. D. Faddeev and B. S. Pavlov. Scattering theory and automorphic functions, Proc. Steklov Inst. Math. 27 (1972), 161-193.

3. P. D. Lax and R. S. Phillips, Scattering theory for automorphic functions, Ann. of Math. Studies, no. 87. Princeton Univ. Press, Princeton, N. J., 1976.

4. The asymptotic distribution of lattice points in Euclidean and non-Euclidean spaces, J. Funct. Anal. 46 (1982), 280-350.

5. Translation representations for automorphic solutions of the wave equation in non-Euclidean spaces. I, II, Comm. Pure Appl. Math. 37 (1984), 303-328, 780-813.

6. S_ Scattering the ory for automorphic functions, Bull. Amer. Math. Soc. 2 (1980), 261-295.

7. A. Selberg, Harmonic analysis and discontinuous groups in weakly symmetric Riemannian spaces with applications to Dirichlet series, J. Indian Math. Soc. 20 (1956), 47-87.

8. A. B. Venkov, Spectral theory of automorphic functions, Proc. Steklov Inst. Math. 153 (1981); English transl., Trudy Mat. Inst. Steklov. (1982).

9. Y. Colin de Verdiere, Pseudo-Laplaciens. II, Ann. Inst. Fourier (Grenoble) 33 (1983), 87-113.

Department of Mathematics, New York University, Courant InStitute of Mathematical SCIENCES, NEW YORK, NEW YORK 10012

Department of Mathematics, Stanford University, Stanford, California 94305 